\title{
Asien - Wachsłumsmärkte für Brandenburger Unternehmen?
}

\author{
Prof. Dr. Bernd Wonneberger, Dipl.-Psych. Hans Könecke, Dipl.-Kfm. (FH) Sven Weickert
}

Erste Ergebnisse des Forschungsprojektes „Markterschließung in Ost- und Südostasien für kleine und mittlere Unternehmen KMU in betrieblicher Kooperation und Erprobung praxisnaher Methoden der Fachhochschulausbildung"

1. Betrachtung der Außenhandelsstatistik Brandenburgs - Schlußfolgerungen für die Zielstellung des Forschungsprojektes

2. Schriftliche Befragung von 2.000 Betrieben

3. Ergebnisse der Firmenbefragung

Literatur

Das Forschungsprojekt läuft vom 1.10.1996 bis 31.10.1997, gefördert durch das Ministerium für Wissenschaft und Kultur des Landes Brandenburg.

\section{Betrachtung der Außenhandelsstatistik Brandenburgs - Schlußfolgerungen für die Zielstellung des Forschungsprojektes}

Ein Ausweis der Leistungsfähigkeit einer Volkswirtschaft ist u.a. die Höhe der Ausfuhren auf den Weltmarkt. Nach der Außenhandelsstatistik des Landesamtes für Datenverarbeitung und Statistik betrugen die Ausfuhren Brandenburgs im Jahr 1991 knapp 2,4 Mrd DM, während sie im letzten erfaßten Jahr 1995 3,5 Mrd DM betrugen.

Ausfuhr des Landes Brandenburg in TDM davon:

\begin{tabular}{l|c|c|c} 
Jahr & Summe & $\begin{array}{c}\text { Ernährungs- } \\
\text { wirtschaft }\end{array}$ & $\begin{array}{c}\text { Gewerbliche } \\
\text { Wirtschaft }\end{array}$ \\
\hline 1991 & 2.376 .572 & 187.772 & 2.188 .800 \\
1992 & 2.603 .991 & 342.630 & 2.261 .361 \\
1993 & 2.502 .050 & 315.770 & 2.186 .280 \\
1994 & 2.820 .082 & 276.504 & 2.543 .578 \\
1995 & 3.541 .731 & 309.184 & 3.232 .547
\end{tabular}

Dieser, auch unter Einrechnung von Preissteigerungen noch erkennbare Anstieg der Exporte, basiert allerdings auf einem vergleichsweise geringen Niveau. Im Jahre 1995 betrugen die gesamten Ausfuhren der Bundesrepublik Deutschland knapp 728 Mrd DM. Somit hatte Brandenburg zuletzt einen Anteil von gerade einmal 0,49 Prozent an den gesamten deutschen Ausfuhren.

Ausfuhranteile am gesamten Außenhandel der BRD in TDM

\begin{tabular}{llllll}
$\begin{array}{l}\text { Jahr Branden- } \\
\text { burg }\end{array}$ & Berlin & $\%$ & $\begin{array}{l}\text { Gesamt- } \\
\text { region }\end{array}$ & BRD gesamt \\
\hline
\end{tabular}

$\begin{array}{llllllll}1993 & 2.502 .050 & 0,40 & 11.391 .616 & 1,81 & 13.893 .666 & 2,21 & 628.386 .638\end{array}$ $\begin{array}{llllllll}1994 & 2.820 .082 & 0.41 & 12.123 .745 & 1,76 & 14.943 .827 & 2,16 & 690.572 .701\end{array}$ $\begin{array}{llllllll}1995 & 3.541 .731 & 0,49 & 11.938 .532 & 1,64 & 15.480 .263 & 2,13 & 727.732 .163\end{array}$

(Quelle: BMWi 1995)
Dieses geringe Exportvolumen der Brandenburger Wirtschaft sollte sicherlich in den nächsten Jahren entscheidend vergrößert werden. Hierfür sollten die vorhandenen Stärken der Brandenburger Wirtschaft ausgebaut werden und noch intensiver als bisher auf dem Weltmarkt bekannt gemacht werden. Den Großteil der Ausfuhren bildeten mit 91,5\% in der Außenhandelsstatistik des Jahres 1995 die Produkte der gewerblichen Wirtschaft. An den gesamten Ausfuhren Brandenburgs von 3,541 Mrd DM hatten die Warengruppen der gewerblichen Wirtschaft folgende Anteile:

$\begin{array}{ll}\text { Rohstoffe } & 2,9 \% \\ \text { Halbwaren } & 16,8 \% \\ \text { Vorerzeugnisse } & 35,7 \% \\ \text { Enderzeugnisse } & 35,9 \%\end{array}$

Demgegenüber betrug der Anteil der Enderzeugnisse (Maschinenbau, Fahrzeuge u.a. Fertigwaren) an den gesamten deutschen Ausfuhren 59,4\% im Jahr 1994.

An dieser Stelle offenbart die Außenhandelsstatistik erneut die strukturellen Probleme der Brandenburger Wirtschaft. Produkte wie Papier und Pappe, Eisenbleche oder andere Vorerzeugnisse können an anderen Standorten auf dem Weltmarkt kostengüinstiger gefertigt werden. Es sind sicher noch erhebliche Anstrengungen der Brandenburger Wirtschaft notwendig, um in den nächsten Jahren:

A) die Marktanteile bei den Vorerzeugnissen auf dem Weltmarkt zu halten, evt. sogar noch auszubauen und vor allem

B) verstärkt Enderzeugnisse, Fertigwaren hoher Qualität für den Weltmarkt zu produzieren.

Welches sind nun die wichtigsten Zielmärkte für die Brandenburger Wirtschaft?

Die Länder Europas sind zu 4/5 die Zielländer bei den Ausfuhren Brandenburgs im Jahr 1995 gewesen. Hierbei ist noch zu erwähnen, daß knapp 1,8 Mrd DM der Ausfuhren Brandenburgs in Mitgliedsländer der EU gingen, dies entspricht einem Anteil der EU-Länder von über 50\% an den gesamten Ausfuhren von 3,5 Mrd DM. Bei der fortschreitenden wirtschaftlichen Integration Europas sind diese strenggenommen gar nicht mehr als Ausfuhren, sondern zukünftig als Binnenhandel zu betrachten.

Bei den Ländern Asiens - seit mehr als 8 Jahren der größte Wachstumsmarkt der Welt - lag allein der Anteil Thailands noch über einem Prozent, alle anderen Länder hatten einen geringeren Anteil. Es erscheint uns offensichtlich, daß die Ausfuhren Brandenburgs in die Länder Asiens mit insgesamt 332 Mio DM derzeit viel zu gering sind im 


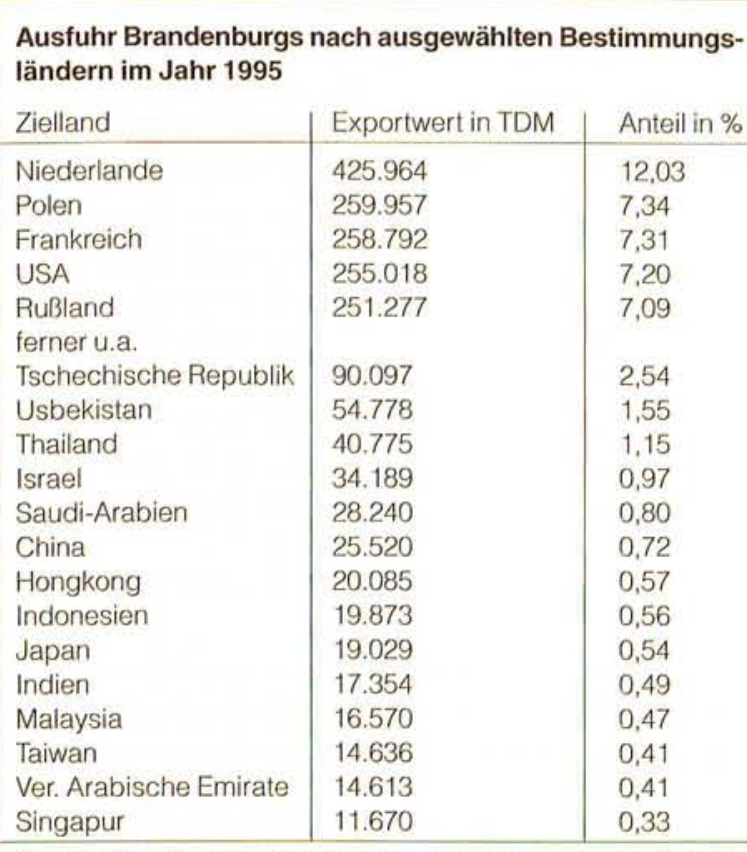

Zum Vergleich: Der Gesamtwert der Exporte 1995 betrug 3.541.731 TDM.

Vergleich zu dem wirtschaftlichen Potential dieser Länder. Genau an diesem Punkt beginnt nun unser Forschungsprojekt, das wir zum 1.10.1996 beginnen konnten. Eine Intensivierung der Wirtschaftsbeziehungen von Brandenburger Klein- und Mittelunternehmen KMU mit den Ländern Ost- und Südostasiens ist das Hauptziel dieses Forschungsprojektes. Neben dem direkten Export, der in der Außenhandelsstatistik mit den Ausfuhren erfasst wird, gibt es natürlich noch andere Strategien der internationalen Markterschließung wie Lizenzverträge, Joint Ventures u. a. mehr (näheres dazu Stahr, 61 ff), die für Brandenburger Unternehmen auf ausländischen Märkten möglich wären. Inwieweit solche Strategien auch zur Sicherung von Arbeitsplätzen in Brandenburg selbst beitragen, ist durch die amtliche Statistik zunächst nicht zu erfassen, so daß wir durch eigene Erhebungen (vgl. Kapitel 2.) herausfinden wollen, welche dieser Strategien zur Erschließung der Märkte in Asien besonders geeignet sind.

Für eine Intensivierung des Brandenburger Außenhandels mit Ost- und Südostasien sehen wir folgende grundsätzliche Möglichkeiten:

A) Gründung oder Ansiedlung von Unternehmen, welche innovative Produkte hoher Qualität auch für den Weltmarkt produzieren können.

B) Ausweitung der internationalen Geschäftstätigkeit bereits existierender Brandenburger Unternehmen. Bei der Realisierung der Strategie A) sind seit Jahren die TINA, die Wirtschaftsförderung Brandenburg GmbH sowie auch private Risikokapital-Funds aktiv, mit denen die TFH Wildau auch zusammenarbeitet. Die Strategie B) ist die Kernaufgabe der BRAHA (jetzt integriert in die Wirtschaftsförderung), mit der wir im Studienschwerpunkt „Internationales Marketing“" seit 1995 eng zusammenarbeiten. Aus unseren Vorstudien und zahlreichen Gesprächen mit unseren Kooperationspartnern und Vertretern der Brandenburger Wirtschaft haben wir letztlich folgende Zielstellungen für unser Forschungsprojekt erarbeitet:

A) Strategien zur erfolgreichen Erschließung ausgewählter Märkte in Südostasien sollen identifiziert werden.
B) Die wichtigsten Probleme bei der Umsetzung der Strategien, wie die Gewinnung von Vertriebspartnern im Zielland, Know-How fuir die Erschließung von Auslandsmärkten, Finanzierung, Rechtsfragen u.a. mehr sollen erfaßt, ggf. Vorschläge zu deren Lösung erarbeitet werden.

C) Durch praxisnahe Methoden der Fachhochschulausbildung wollen wir gut qualifizierte und hoch motivierte Absolventen zur Erschließung dieser Märkte ausbilden fuir Betriebe, die Schritt nach Asien planen.

In den folgenden Abschnitten soll der Stand unseres Forschungsprojektes Anfang Februar 1997 dargestellt werden.

\section{Schriftliche Befragung von 2.000 Betrieben}

Wir wollten $2.000 \mathrm{KMU}$ im Rahmen einer schriftlichen Befragung anschreiben mit mehr als 10 und weniger als 250 Mitarbeitern. Die untere-Grenze von 10 Beschäftigten - die diskussionsfähig ist - legten wir mit der Überlegung fest, daß für eine erfolgreiche Auslandsmarkterschließung in den ASEAN-Staaten Kleinstunternehmen mit weniger als 10 Beschäftigten nur in wenigen Fällen die innerbetrieblichen Ressourcen besitzen, selbst wenn das Produkt innovativ ist. Nach oben hin haben wir uns entschlossen, bei der geringen Anzahl von Großunternehmen in der Region, auch diese mit zu befragen (und ggf. deren Antworten herauszurechnen).

Als Wirtschaftszweige wurden ausgewählt:
A) Gewerbliche Wirtschaft
SICode 21 bis 37
B) EDV-Dienstleistungen
SICode 72
C) andere Dienstleistungen
SICode 73
D) Ingenieurbüros
SICode 74

Die zuletzt in B. - D. genannten Branchen wurden ausgewählt, weil auch in diesen KMU ein weltmarktfähiges, technologisches Know-How vermutet werden kann (vgl. dazu Kulicke).

Die Adressen der zu befragenden KMU wollten wir von den Industrie- und Handelskammern Brandenburgs beziehen, da deren Mitgliedsadressen laufend aktualisiert werden durch die Gewerbean- bzw. abmeldungen. Anfragen beim Adressenservice der IHK zeigten dann, daß in den genannten Wirtschaftszweigen nur etwa 900 Unternehmen in Brandenburg in den geannten Branchen registriert sind. Deswegen entschieden wir uns, ebenfalls Berliner KMU zu befragen, wodurch ja das Spektrum der tatsächlich praktizierten Strategien gemäß unserer Zielstellung A: (vgl. Kapitel 1.) auch erweitert wird. Bei Vorstudien durch Projekt- und Diplomarbeiten unserer Studenten und Studentinnen hatten wir bsw. kleinere Unternehmen der Berliner Elektrotechnik kennengelernt, die bereits mit 12 Mitarbeitern mehrere Mio DM Exportumsatz erzielten. Sofern also die Strategien dieser exportorientierten Unternehmen verallgemeinerbar sind für andere Wirtschaftszweige, hofften wir, auch in Berlin 
Unternehmen zu finden, die Vorreiter- und Vorbildfunktionen auf internationalen Märkten für andere Unternehmen der Region Berlin-Brandenburg ausüben könnten.

Letztlich erhielten wir 269 auswertbare Fragebögen zurück, worüber nunmehr die ersten Auswertungen vorliegen, die wir im folgenden Kapitel darstellen wollen.

\section{Ausgewählte Ergebnisse der Firmen- befragung}

Von den befragten 269 Unternehmen gaben 86 an, sie seien in Europa tätig; 98 gaben an, sie seien international tätig. Ihr Interesse an internationalen Geschäftsbeziehungen bezeichneten als „mittel“ 49 Unternehmen, als „groß“ 87 Unternehmen und als „sehr groß“ 75 Unternehmen.

Mit der Formulierung „Unsere Geschäftsbeziehungen würden wir am ehesten ausweiten nach..." erfragten wir dann, welche Ländermärkte die Unternehmen jeweils für interessant hielten. Die folgende Tabelle gibt die Anzahl der Nennungen wieder, wobei Mehrfachnennungen möglich waren:

\begin{tabular}{lr} 
Länder & Anzahl \\
\hline Europa/Europäische Union & 151 \\
Osteuropa und GUS-Staaten & 148 \\
Indonesien, Thailand, Taiwan, Singapur, & \\
Malaysia, Korea (ASEAN) & 68 \\
China & 58 \\
Kanada, Mexiko, USA (NAFTA) & 53 \\
Lateinamerika & 37 \\
Japan & 31 \\
arabischer Raum & 30 \\
Indien & 25 \\
Afrika & 18 \\
andere Länder & 4
\end{tabular}

Die Priorität der Ländermärkte in dieser Stichprobe Berliner und Brandenburger KMU ähnelt in einigen Ländern den Forschungsergebnissen anderer Autoren (vgl. Holzmüller, Köglmayr und Müller). Dichtl, Köglmayr und Müiller entwikkelten in den 80er Jahren einen umfangreichen Test zur Auslandsorientierung von Managern. Dieser Test wurde mit den Managern von KMU durchgefuihrt in Deutschland, Österreich, Finnland, Südafrika, Japan und Korea. Mit einem umfangreichen Instrumentarium maßen sie die „kognitive Distanz“ der befragten Manager zu ausgewählten Ländermärkten. Den Begriff der „kognitiven Distanz“ sowie die Methode ihrer Bestimmung wollen wir hier nicht weiter diskutieren. Aus deutscher Sicht betrug die gemessene Distanz zu den USA das 3fache der Distanz zu den Niederlanden, zu Japan und Hongkong das 8fache der Distanz zu den Niederlanden (vgl. Müller, 168ff).

In den oben genannten Ergebnissen unserer schriftlichen Befragung finden wir diese große Distanz zu Asien nicht wieder. Das Interesse vieler Unternehmen an den asiatischen Märkten hat zugenommen, was durch die hohe Attraktivität und verbesserte Erreichbarkeit dieser Märkte zu erklären ist. Sowohl die ASEAN-Staaten wie China erhielten in unserer Befragung mehr Nennungen als die NAFTA-Staaten (Kanada, Mexiko, USA). Vermutlich haben jedoch vor allem jene KMU den Fragebogen zurüickesandt, welche ihre internationalen Aktivitäten in Richtung Asien entwickeln wollen (=Positivauswahl der Stichprobe). Im Laufe unserer weiteren Forschungen wollen wir u.a. auch versuchen, die „kognitive Distanz" der Manager in Berlin und Brandenburg durch persönliche Interviews näher zu bestimmen.

Trotz ihrer Größe und Stärke sind die Wirtschaftsregionen Nordamerika/NAFTA und Ostasien/Pazifik für die Berliner und Brandenburger Unternehmen nur ,zweite Wahl“. Hierfür sehen wir folgende Erklärungen, die wir als Hypothesen für unsere weiteren Forschungen formulierten:

1. Die „kognitive Distanz“ zu diesen Märkten ist relativ groß.

2. Die Kosten der Markterschließung in Nordamerika/ NAFTA oder Ostasien/Pazifik übersteigen die Finanzkraft kleiner und mittlerer Unternehmen (vgl. BMWI 1996b, 19).

3. Es fehlt vielen Betrieben das Know-How für eine Erschließung dieser Märkte.

4. Neben nicht ausreichend wahrgenommener Beratung fehlt es auch an entsprechend ausgebildetem und erfahrenem Personal.

Um die „kognitive Distanz“ der deutschen KMU gegenüber außereuropäischen Märkten zu verringern, muissen vielfältige Länderinformationen über Sitten und Gebräuche, ökonomische und modische Trends, Gewohnheiten der privaten Verbraucher und anderer Abnehmer, Rechtsauskünfte u.a. mehr durch die Leitung eines KMU gesammelt, gewichtet und in die Unternehmensstrategie eingebunden werden. An dieser Stelle zeigt sich einmal mehr ein typischer Engpaß bei der Führung von KMU: Die Unternehmensstragie wird zu großen Teilen allein von dem oder den Inhabern definiert, welcher wiederum selbst die für strategische Entscheidungen notwendigen Informationen sammelt und gewichtet (vgl. Mugler, 20f). Letztlich entstehen so für den Inhaber eines KMU „Zeit oder Opportunitätskosten" der Beschaffung von Informationen über Auslandsmärkte (BMWI 1996b, 18). Im Rahmen unserer schriftlichen Befragung wollten wir wissen, welche Quellen die KMU nutzen zur Information über die asiatischen Märkte, worüber wir folgende Nennungen erhielten:

\begin{tabular}{lr} 
Quelle & Anzahl \\
\hline Fachzeitschriften & 157 \\
Tageszeitung & 134 \\
Fernsehen & 107 \\
Kontakte zu anderen Unternehmen & 90 \\
IHK-Außenwirtschaftsabteilungen & 87 \\
Publikationen der Fachverbände & 84 \\
Bundesstelle für Außenhandels-Informationen & 63 \\
Veröffentlichungen der Kreditinstitute & 47 \\
Veröffentlichungen kommerzieller & \\
Marktforschungsinstitute & 43 \\
Ostasienausschuß dt. Wirtschaft & 21 \\
sonstige Quellen & 17 \\
Publikationen Hochschulen & 13
\end{tabular}

Es ist bemerkenswert, daß erst an fünfter bzw. siebter Stelle jene Institutionen der gewerblichen Wirtschaft bzw. 
des Wirtschaftsministeriums genannt werden, welche das Sammeln und Bereitstellen von Länderinformationen zur Kernaufgabe haben. Vorsichtig interpretierend können wir sagen: Die Nutzung von Tagespresse und Fachzeitschriften auch zur Information über die asiatischen Märkte scheint für die Inhaber von KMU zeitökonomischer zu sein als eine Anfrage bei der IHK oder bei der BfAI.

Wir teilen von daher die Schlußfolgerung des BMWi: „Erforderlich erscheint uns, die Wahrnehmungsschwelle zu senken, indem der aktiven Informations-Vermittlung und Kontaktanbahnung zu Unternehmen (Hervorhebung in der Quelle) Vorrang vor der passiven Informationsbereitstellung eingeräumt wird“" (BMWi 1996b, 18).

Es wäre allerdings Gegenstand eines eigenen Forschungsprojektes zu untersuchen, welche Formen dieser aktiven Informationsvermittlung und Kontaktanbahnung tatsächlich ökonomisch effektiv sind, d. h. die Geschäftsbeziehungen zwischen deutschen und asiatischen Partnern initiieren und stabilisieren können.

Allerdings erwarten wir nicht, daß allein neue Formen der Information und Kontaktanbahnung den Einstieg von Berliner und Brandenburger KMU in asiatische Märkte nachhaltig auslösen würden.

Wir wollten von den befragten Unternehmen wissen, was sie bei der Erschließung internationaler Märkte für völlig problemlos oder andererseits auch für extrem schwierig hielten. Dies sind die Extremwerte eines sechsstufigen Rankings, das die KMU für 13 Probleme der Markterschließung als Einschätzung vergeben konnten. Zur besseren Übersichtlichkeit sind die Rankings 1 (völlig problemlos) - 3 (mittlerer Schwierigkeitsgrad) als „lösbares Problem“ und die Rankings 4 (nicht so einfach zu lösen) - 6 (extrem schwierig) als „ziemlich kompliziertes Problem " mit der Summe der gewichteten Punktwerte in der folgenden Darstellung zusammengefasst.

\begin{tabular}{lcc} 
Probleme der Markterschließung & $\begin{array}{c}\text { gut } \\
\text { lösbar }\end{array}$ & $\begin{array}{c}\text { ziemlich } \\
\text { kompliziert }\end{array}$ \\
\hline $\begin{array}{l}\text { Kontakte zu Kunden } \\
\text { Währungsstabilităt }\end{array}$ & 122 & 115 \\
Gewinnung qualifizierter & 136 & 85 \\
Mitarbeiter & & \\
Steuern/Gewinntransfer & 116 & 97 \\
Kontakte zu Vertriebspartnern & 116 & 77 \\
Kontakte zu Lieferanten & 117 & 106 \\
Sprache & 148 & 50 \\
Landeskultur & 167 & 61 \\
Finanzierung & 176 & 36 \\
Zölle & 93 & 125 \\
politische Rahmenbedingungen & 131 & 80 \\
verschiedene Rechtssysteme & 133 & 84 \\
\end{tabular}

Man kann feststellen, daß eigentlich alle genannten Probleme von der Mehrheit der befragten KMU als „ösbar“ eingestuft werden; einzig die Finanzierung wird bei der Erschließung internationaler Märkte als „ziemlich kompliziert" wahrgenommen. Dementsprechend hielten bei den Anworten auf eine weitere Frage in unserer Umfrage eine Unterstützung bei der Finanzierung 90 der Befragten für ziemlich wichtig, 79 der Befragten sogar am wichtigsten.
Letztlich werden Fragen der Finanzierung internationaler Aktivitäten von Berliner und Brandenburger KMU ein Thema unseres Wildauer Workshops sein, den wir mit interessierten Unternehmen, Banken und Vertretern der Fachverbände im Oktober 1997 veranstalten werden. Weitere Ergebnisse unseres Forschungsprojektes, auch aus Experteninterviews, werden wir bei diesem Workshop vorstellen.

\section{Liferatur}

BMWi Bundesministerium für Wirtschaft (1995): Wirtschaft in Zahlen '95; Bonn

BMWi Bundesministerium für Wirtschaft (1996a): Exportfibel - Wegweiser für kleine und mittlere Unternehmen; Bonn

BMWi Bundesministerium für Wirtschaft (1996b): Investitionschancen und Erfahrungen kleiner und mittlerer Unternehmen im asiatisch-pazifischen Raum; Bonn

Holzmuiller, Hartmut H. (1995):

Konzeptionelle und methodische Probleme in der interkulturellen Management- und Marketingforschung; Stuttgart

Kulicke, Marianne (1993):

Chancen und Risiken junger Technologieunternehmen; Heidelberg

Köglmayr, Hans-Georg (1990):

Die Auslandsorientierung von Managern als strategischer Erfolgsfaktor; Berlin

Müller, Stefan (1991):

Die Psyche des Managers als Determinante des Exporterfolges; Stuttgart

Mugler, Josef (1995):

Betriebswirtschaftslehre der Klein- und Mittelbetriebe;

2. Auflage, Wien und NewYork

Stahr, Gunter (1993):

Internationales Marketing; Ludwigshafen (Rhein)

\section{Autoren}

Prof. Dr. Bernd Wonneberger

Dipl.-Psych. Hans Könecke

Dipl.-Kfm. (FH) Sven Weickert

Technische Fachhochschule Wildau

Institut für Internationales Marketing und Unternehmensführung

Fachbereich Wirtschaft, Verwaltung und Recht

Tel. $\quad++49(0) 3375-508325$

Fax $++49(0) 3375-508388$ 